\title{
Chemotherapy for metastatic colon cancer: No effect on survival when the dose is reduced due to side effects
}

\author{
Stefan Munker ${ }^{1 *}$, Michael Gerken ${ }^{2}$, Petra Fest ${ }^{3}$, Claudia Ott ${ }^{1}$, Elisabeth Schnoy ${ }^{1}$, Stefan Fichtner-Feigl ${ }^{4}$, \\ Philipp Wiggermann ${ }^{5}$, Martin Vogelhuber ${ }^{6}$, Wolfgang Herr ${ }^{6}$, Christian Stroszczynski ${ }^{5}$, Hans Jürgen Schlitt ${ }^{4}$, \\ Matthias Evert ${ }^{7}$, Michael Reng ${ }^{8}$, Monika Klinkhammer-Schalke ${ }^{2}$ and Andreas Teufel ${ }^{1 *}$
}

\begin{abstract}
Background: 5-Fluorouracil (5FU), Folinic acid (FA), and Oxaliplatin (FOLFOX) or 5FU, FA, and Irinotecan (FOLFIRI) are standard regimens for palliative chemotherapy of metastatic colon cancer. Since data showing the influence of dose reduction in palliative treatment are rare, the objective of this single center, retrospective study was to further characterize the influence of dose reduction on efficacy of these therapeutic regimens.

Methods: One hundred nine patients, diagnosed with stage IV colon cancer between 2004 and 2012 and receiving palliative first-line chemotherapy with either FOLFOX or FOLFIRI regimens in our outpatient clinic were analyzed for treatment efficacy. Patients who received dose reductions due to side effects usually received doses of $80 \%$ or lower of per protocol dose. Survival data were obtained from the Regensburg Tumor Registry. Survival analysis was performed using Kaplan-Meier statistical analysis and multivariable analysis.

Results: A dose reduction due to side effects was necessary in 46 (42\%) patients. Dose reduction was independent of age. Major reasons for dose reduction were neutropenia (30\%) followed by polyneuropathy (16\%) and diarrhea (14\%). Dosage was more often reduced in patients receiving FOLFOX based therapy. Comparison of patients with dose reduction versus patients with full dosage showed no significant difference on overall survival $(p=0.430)$. Subgroup analysis revealed dose reduction in patients with N2 stage disease was associated with improved survival. Patients who underwent dose reduction received more cycles of chemotherapy (13.7 vs. 10.8 cycles) and cumulative dosage was similar in both groups.

Conclusion: Contrary to our expectations, the need to reduce chemotherapy dosage due to side effects does not indicate a worse prognosis in our retrospective analysis. We believe this can in part be explained by better adaption to interindividual pharmacokinetics and longer time of treatment.
\end{abstract}

Keywords: Dose reduction, Cancer, Colorectal cancer, Chemotherapy

\section{Background}

Colon cancer is the third most common cancer and a major cause of morbidity and mortality worldwide [1]. During the last few decades, improvement in therapeutic regimens for advanced colorectal cancer led to a dramatic increase in efficacy, reduction of mortality rates,

\footnotetext{
* Correspondence: stefan.munker@klinik.uni-regensburg.de; andreas.teufel@klinik.uni-regensburg.de

'Department of Internal Medicine I, University Hospital Regensburg,

Franz-Josef-Strauss Allee 11, 95053 Regensburg, Germany

Full list of author information is available at the end of the article
}

and improved survival. Upon diagnosis, $20 \%$ of newly diagnosed colorectal cancer patients present with metastatic disease with no curative treatment options currently available. Among the chemotherapy regimens considered effective in palliative treatment, Irinotecan or Oxaliplatin in combination with 5-Fluorouracil regimens are standard back bones of current systemic treatment $[2,3]$.

These chemotherapy regimens were initially tested for efficacy in well-defined study populations not necessarily reflecting average (multimorbid older) patients in real

(c) The Author(s). 2018 Open Access This article is distributed under the terms of the Creative Commons Attribution 4.0 International License (http://creativecommons.org/licenses/by/4.0/), which permits unrestricted use, distribution, and reproduction in any medium, provided you give appropriate credit to the original author(s) and the source, provide a link to the Creative Commons license, and indicate if changes were made. The Creative Commons Public Domain Dedication waiver (http://creativecommons.org/publicdomain/zero/1.0/) applies to the data made available in this article, unless otherwise stated. 
life settings [4, 5]. Clinicians increasingly realize the shortcoming of the initial studies [6] since these mostly included younger patients better able to cope with adverse side effects or toxicities [7]. But especially in elderly and comorbid patients, side effects, organ toxicities and therefore potentially limited survival need to be considered [8]. In clinical practice, these effects are prevented or mitigated by a dose reduction of chemotherapy with the suspected consequence of worse tumor related survival [9]. We therefore believe that there is a need to further investigate the impact of dose reduction of the currently used therapeutic "standard" regimens on survival and side effects in different subgroups. Hence, we performed a retrospective analysis of our patient cohort with advanced stage colorectal cancer patients to assess outcome of reduced chemotherapy dosage.

\section{Methods}

\section{Study design}

A retrospective analysis was performed based on clinical data obtained from the population-based clinical cancer registry at the Regensburg Tumor Center in Eastern Bavaria, Germany. Epidemiological data and survival were investigated in a cohort of colon cancer diagnosed between 2004 and 2012 receiving chemotherapy in the outpatient clinic of the University Medical Center Regensburg. Patients with stage IV colorectal cancer undergoing palliative combination chemotherapy were divided into dose reduction $(\leq 80 \%)$ and full dosage (100\%) groups. We intend to analyze survival time by Kaplan Meier method and to estimate 2- and 3-year survival rates and mean and median survival time. Data collection and retrospective analysis of patient information were anonymized in accordance with the Declaration of Helsinki, and in line with the Bavarian Law of Cancer Registration.

\section{Background and data collection}

The baseline cohort of the present study consisted of patients with the ICD-10-GM (http://www.dimdi.de/static/ de/klassi/icd-10-gm/index.htm) diagnosis C18, i.e. a malignant neoplasm of colon. 109 patients with advanced stage colon carcinoma (UICC Stage IV) with histologically confirmed adenocarcinoma of the colon between 2002 and 2012 receiving palliative chemotherapy in our University Medical Center were included in the study. Neuroendocrine tumors were excluded. 3 Patients receiving only $5 \mathrm{FU}$ based chemotherapy regimens without Irinotecan or Oxaliplatin were excluded. Prior to palliative chemotherapy, 72 patients received palliative or oncological tumor resection. In this subgroup, the diagnosis of metastasized colon cancer was made during surgery or in follow up examinations. Patients were routinely assessed prior to chemotherapy by a physician trained in oncology. Efficacy evaluation was performed by CT scans in 3-month intervals. Patients who died of cancer unrelated causes and patients with death within one month after start of palliative chemotherapy were censored.

Baseline characteristics are shown in Table 1. For assessing comorbidities a scoring according to the Charlson Score comorbidities index [10] was performed Additional file 1: Table S1. Patients receiving either FOLFOX, FOLFIRI or sequentially both chemotherapy regimens were included in this study. Mode of administration was exclusively i.v.. Chemotherapy dosing values were calculated and registered with a chemotherapy planning software $\left(\mathrm{OnkoDAT}^{\bullet}\right)$ [11]. A patient was considered receiving reduced dosage of chemotherapy if he received $\geq 3$ cycles of less than $80 \%$ of $5 \mathrm{FU}$, Oxaliplatin, or Irinotecan. Survival information were obtained from the Regensburg Tumor Center founded in 1991. The Cancer registry includes epidemiological and clinical data from all consenting patients with malignancies diagnosed and treated in Eastern Bavaria (2.1 million population). All data were extracted, recorded, and fed into a central database by trained personnel. The patients' life status and disease recurrence were ascertained from clinical reports, death certificates issued by the local public health departments, and the registration offices of the respective residential districts. Data were processed and secured according to the Bavarian Law of Cancer Registration.

\section{Statistical analysis}

Continuous data were described as means, median, minimum, maximum values and standard deviation, and categorical data were expressed as absolute frequencies and relative percentages. Patient characteristics were compared with t-tests for normally distributed continuous data, otherwise by means of non-parametric Mann-Whitney-U- and Chi-square tests for categorical variables. Tumor-specific survival rates (OS) were analyzed from the start of palliative chemotherapy until the event of death or last patient contact. Survival rates of patients with and without dose reduction were described by Kaplan-Meier analysis. Survival differences were tested for statistical significance by the two-sided Log Rank in Kaplan-Meier analyses; the level of significance was set to 0.05 . The follow-up period and survival times were right censored using December 31, 2012 as cut-off date. To determine the influence of further co-variables on overall survival, we performed univariable and multivariable regression analysis using Cox proportional hazard models. In multivariable analysis, the hazard ratio (HR) was adjusted for the co-variables age, sex, TNM status, grading, lymph vessel invasion, vein invasion, Charlson score for comorbidities, surgery and 
Table 1 Patient characteristics according to dosage reduction and in total

\begin{tabular}{|c|c|c|c|c|c|c|c|c|}
\hline & & \multicolumn{6}{|c|}{ Dosage reduction } & \multirow{3}{*}{$\begin{array}{l}\text { T-test } \mathrm{Chi}^{2} \\
p \text { value }\end{array}$} \\
\hline & & \multicolumn{2}{|c|}{ Yes ( $<=80 \%$ dosage) } & \multicolumn{2}{|c|}{ No (=100\% dosage) } & \multicolumn{2}{|c|}{ Total } & \\
\hline & & N & $(\%)$ & N & (\%) & N & $(\%)$ & \\
\hline \multirow[t]{3}{*}{ Age } & Mean/Median & 56.5 & 59.0 & 58.4 & 58.0 & 57.6 & 59.0 & .440 \\
\hline & $<65$ & 32 & $69.6 \%$ & 42 & $66.7 \%$ & 74 & $67.9 \%$ & .749 \\
\hline & $>=65$ & 14 & $30.4 \%$ & 21 & $33.3 \%$ & 35 & $32.1 \%$ & \\
\hline \multirow[t]{2}{*}{ Sex } & Male & 29 & $63.0 \%$ & 46 & $73.0 \%$ & 75 & $68.8 \%$ & .267 \\
\hline & Female & 17 & $37.0 \%$ & 17 & $27.0 \%$ & 34 & $31.2 \%$ & \\
\hline \multirow[t]{3}{*}{ T stage } & T3 & 17 & $37.0 \%$ & 23 & $36.5 \%$ & 40 & $36.7 \%$ & \\
\hline & T4 & 22 & $47.8 \%$ & 22 & $34.9 \%$ & 44 & $40.4 \%$ & .205 \\
\hline & Tx & 7 & $15.2 \%$ & 18 & $28.6 \%$ & 25 & $22.9 \%$ & \\
\hline \multirow[t]{4}{*}{ N stage } & No & 4 & $8.7 \%$ & 5 & $7.9 \%$ & 9 & $8.3 \%$ & \\
\hline & N1 & 14 & $30.4 \%$ & 16 & $25.4 \%$ & 30 & $27.5 \%$ & .796 \\
\hline & N2 & 18 & $39.1 \%$ & 23 & $36.5 \%$ & 41 & $37.6 \%$ & \\
\hline & $\mathrm{Nx}$ & 10 & $21.7 \%$ & 19 & $30.2 \%$ & 29 & $26.6 \%$ & \\
\hline \multirow[t]{3}{*}{ Grading } & G2 & 27 & $58.7 \%$ & 40 & $63.5 \%$ & 67 & $61.5 \%$ & \\
\hline & G3 & 18 & $39.1 \%$ & 19 & $30.2 \%$ & 37 & $33.9 \%$ & .419 \\
\hline & Gx & 1 & $2.2 \%$ & 4 & $6.3 \%$ & 5 & $4.6 \%$ & \\
\hline \multirow[t]{3}{*}{ L stage } & LO & 6 & $13.0 \%$ & 7 & $11.1 \%$ & 13 & $11.9 \%$ & \\
\hline & L1 & 23 & $50.0 \%$ & 15 & $23.8 \%$ & 38 & $34.9 \%$ & .010 \\
\hline & Lx & 17 & $37.0 \%$ & 41 & $65.1 \%$ & 58 & $53.2 \%$ & \\
\hline \multirow[t]{3}{*}{ V stage } & V0 & 13 & $28.3 \%$ & 11 & $17.5 \%$ & 24 & $22.0 \%$ & \\
\hline & V1 & 14 & $30.4 \%$ & 10 & $15.9 \%$ & 24 & $22.0 \%$ & .030 \\
\hline & $V x$ & 19 & $41.3 \%$ & 42 & $66.7 \%$ & 61 & $56.0 \%$ & \\
\hline \multirow[t]{5}{*}{ Charlson Comorbidity Score } & 6 & 32 & $69.6 \%$ & 44 & $69.8 \%$ & 76 & $69.7 \%$ & \\
\hline & 7 & 7 & $15.2 \%$ & 13 & $20.6 \%$ & 20 & $18.3 \%$ & \\
\hline & 8 & 6 & $13.0 \%$ & 4 & $6.3 \%$ & 10 & $9.2 \%$ & .336 \\
\hline & 9 & 0 & $0.0 \%$ & 2 & $3.2 \%$ & 2 & $1.8 \%$ & \\
\hline & 10 & 1 & $2.2 \%$ & 0 & $0.0 \%$ & 1 & $0.9 \%$ & \\
\hline \multirow[t]{2}{*}{ Oncological resection/Surgery } & Yes & 35 & $76.1 \%$ & 37 & $58.7 \%$ & 72 & $66.1 \%$ & .059 \\
\hline & No & 11 & $23.9 \%$ & 26 & $41.3 \%$ & 37 & $33.9 \%$ & \\
\hline \multirow[t]{5}{*}{ No. of CTX lines } & 1 & 11 & $23.9 \%$ & 17 & $27.0 \%$ & 28 & $25.7 \%$ & \\
\hline & 2 & 23 & $50.0 \%$ & 34 & $54.0 \%$ & 57 & $52.3 \%$ & \\
\hline & 3 & 11 & $23.9 \%$ & 11 & $17.5 \%$ & 22 & $20.2 \%$ & .587 \\
\hline & 4 & 0 & $0.0 \%$ & 1 & $1.6 \%$ & 1 & $0.9 \%$ & \\
\hline & 5 & 1 & $2.2 \%$ & 0 & $0.0 \%$ & 1 & $0.9 \%$ & \\
\hline \multirow[t]{2}{*}{ Biological } & Yes & 21 & $45.7 \%$ & 26 & $41.3 \%$ & 47 & $43.1 \%$ & .648 \\
\hline & No & 25 & $54.3 \%$ & 37 & $58.7 \%$ & 62 & $56.9 \%$ & \\
\hline \multirow[t]{5}{*}{ CTX regimen sequence } & FOLFOX-> FOLFIRI & 30 & $65.2 \%$ & 27 & $42.9 \%$ & 57 & $52.3 \%$ & \\
\hline & FOLFIRI- > FOLFOX & 3 & $6.5 \%$ & 13 & $20.6 \%$ & 16 & $14.7 \%$ & .044 \\
\hline & FOLFOX & 10 & $21.7 \%$ & 13 & $20.6 \%$ & 23 & $21.1 \%$ & \\
\hline & FOLFIRI & 3 & $6.5 \%$ & 10 & $15.9 \%$ & 13 & $11.9 \%$ & \\
\hline & Total & 46 & $100.0 \%$ & 63 & $100.0 \%$ & 109 & $100.0 \%$ & \\
\hline
\end{tabular}


further chemotherapy, i.e. number of lines and regimens. Again, a two-sided $p$-value of 0.05 was considered to indicate statistical significance. Hazard ratios and corresponding 95\% confidence intervals (CI) were calculated and considered statistically significant when the CI excluded 1 . 0 . All analyses were performed using IBM SPSS Statistics, version 23.0.

\section{Results}

\section{Reduction of chemotherapy dosage}

Among 109 patients 67\% (73/109) of the patients received both (FOLFOX and FOLFIRI) regimens sequentially. The majority $78 \%(57 / 73)$ began with FOLFOX and changed during the course of disease to FOLFIRI, $21 \%(23 / 109)$ of the patients received exclusively FOLFOX, whereas FOLFIRI chemotherapy regimens were used exclusively in 12\% (13/109) of patients. 43\% (47/109) received additional treatment with a biological agent.

Upon discretion of the treating physician, in most cases dosage was reduced to $80 \%$ of the protocol dosage. We therefore used reduction to $80 \%$ of protocol dosage as a cut off to investigate the clinical effectiveness of standard chemotherapy in patients receiving reduced dosage.

In $42 \%(46 / 110)$ of our patients a dose reduction to $80 \%$ or less was performed during the course of chemotherapy. Those 46 patients who received a dose reduction, received in average 14 chemotherapy cycles in our clinic (Mean: 13.7 cycles; +-12.3 cycles). Patients continuing on protocol chemotherapy doses, received 10.5 cycles (stdv 10.8, $p=0.149$ ). In more detail, the majority of chemo cycles (72\%, median: $81 \%$; stdv: $27 \%$ ) in these patients were applied with reduced dosage. Average cumulative dosage and dose intensities for 5Flourouracil, Irinotecan and Oxaliplatin were calculated for full dosage and dose reduction subgroups (Table 2).
As expected, relative dose intensities were significantly different. Even though a trend to a higher cumulative dosage can be observed, t-test revealed no significant differences for cumulative dosages.

\section{Dose reduction in subgroups - analysis of distribution}

In preparation to compare survival rates, we compared the distribution of the co-variables age, sex, TNM status, grading, lymph vessel invasion, vein invasion, Charlson score for comorbidities, surgery and further chemotherapy, i.e. number of lines and regimens in the dose reduction and full dosage group.

No significant difference was seen except for lymph vessel and vein invasion, CTX regimen and adverse side effects, when Chi-square test for independence was performed. As expected, subgroups of patients suffering from severe symptoms/side effects related to chemotherapy such as diarrhea $(p<0.001)$, PNP $(p<0.001)$, or neutropenia $(p=0.02)$ received dose reduction almost exclusively. Detailed summary of the distribution of dose reductions in different subgroups is given in Table 1.

\section{Reasons for dose reduction}

The most common reason for dose reduction of chemotherapy was neutropenia (30\%). Other common side effects leading to dose reduction were polyneuropathy $(16 \%)$ and diarrhea (14\%). Polyneuropathy was generally due to treatment with Oxaliplatin (10 patients, 14\%). Diarrhea was observed at similar rates in both Oxaliplatin $(6 / 48$ patients, $13 \%)$ and Irinotecan treated patients (4/30 patients, 13\%). Less common reasons for dose reduction included mostly symptom-related causes, hyperemesis, worsening of general condition, hand foot syndrome and mucositis. A complete overview of the reasons for dose reduction of chemotherapy is shown in Table 3.

Table 2 Dose intensity and cumulative dosage for the dose-reduction and full dosage subgroups

\begin{tabular}{|c|c|c|c|c|c|}
\hline & Substance & Dose group & Mean in mg & standard deviation & $p$-value \\
\hline \multirow[t]{6}{*}{ Cumulative Dosage } & \multirow[t]{2}{*}{ 5-Fluorouracil } & $100 \%$ & 54,934 & 55,617 & \multirow[t]{2}{*}{0.42} \\
\hline & & $<80 \%$ & 64,321 & 61,824 & \\
\hline & \multirow[t]{2}{*}{ Oxaliplatin } & $100 \%$ & 1046 & 852 & \multirow[t]{2}{*}{0.34} \\
\hline & & $<80 \%$ & 1245 & 976 & \\
\hline & \multirow[t]{2}{*}{ Irinotecan } & $100 \%$ & 2861 & 3295 & \multirow[t]{2}{*}{0.77} \\
\hline & & $<80 \%$ & 3099 & 3296 & \\
\hline \multirow[t]{6}{*}{ Dose intensity } & \multirow[t]{2}{*}{ 5-Fluorouracil } & $100 \%$ & 5383 & 1450 & \multirow[t]{2}{*}{0.002} \\
\hline & & $<80 \%$ & 4547 & 1178 & \\
\hline & \multirow[t]{2}{*}{ Oxaliplatin } & $100 \%$ & 173 & 31 & \multirow[t]{2}{*}{0.03} \\
\hline & & $<80 \%$ & 156 & 35 & \\
\hline & \multirow[t]{2}{*}{ Irinotecan } & $100 \%$ & 311 & 74 & \multirow[t]{2}{*}{0.04} \\
\hline & & $<80 \%$ & 274 & 65 & \\
\hline
\end{tabular}

$P$-values were calculated with t-test 
Table 3 Reasons for dose reduction

\begin{tabular}{lll}
\hline Distribution of adverse effects & $N$ & $\%$ \\
\hline Leukopenia & 20 & $30 \%$ \\
Polyneuropathy & 11 & $16 \%$ \\
Diarrhea & 10 & $14 \%$ \\
Worsening of general condition & 6 & $9 \%$ \\
Hand-foot-syndrome & 5 & $7 \%$ \\
Thrombocytopenia & 5 & $7 \%$ \\
Hyperemesis & 3 & $4 \%$ \\
Elevated bilirubin & 2 & $3 \%$ \\
Deterioration of kidney function & 1 & $1 \%$ \\
Mucositis & 1 & $1 \%$ \\
Not specified & 5 & $7 \%$ \\
\hline
\end{tabular}

\section{Survival in dose reduced versus full dose 5-FU based regimens}

In terms of tumor-specific survival, we did not observe any differences between patients receiving full dose and reduced dose chemotherapy (Log Rank, $p=0.430$ ) (Fig. 1). Median survival for patients receiving full dosage was 13 . 0 months (Mean 19.1), for patients with dosage reduction 14.9 months (Mean 21.2). Two-year survival was $19.5 \%$ (full dosage) vs $35.8 \%$ (reduced dosage). Three-year survival rate of patients with full dose and reduced dose chemotherapeutic treatment was $19.5 \%$ and $9.1 \%$. A univariable Cox regression rendered a hazard ratio of 0.841 (95\% CI $0.547-1.294 ; p=0.431$ ) for the dose reduction group versus full reduction. After adjustment for age, sex, TNM status, grading, lymph vessel invasion, vein invasion,
Charlson score for comorbidities, surgery and further chemotherapy, i.e. number of lines and regimens in a multivariable Cox regression analysis the hazard ratio for patients with dose reduction was 0.861 (95\% CI 0.492-1.506; $p=0.600)$ Table 4 .

\section{Efficacy - subgroup analysis}

We performed a subgroup analysis to identify potential subgroups in which reduction of chemotherapy might be beneficial or harmful. Table 5 summarizes survival in several subgroups with respect to dose reduction. In most subgroups, no significant differences in survival were observed. Unexpectedly, in the stage N2 lymph node subgroup with 41 patients dose reduction was associated with improved survival (Log Rank $p=0.024)$.

\section{Discussion}

Over the past decades a considerable progress has been made in the management of colorectal cancer by medical oncologists. The studies leading to these advances were predominantly performed on young and healthy populations; hence the common practice of dose reduction in elderly or frail patients was not a primary issue. Thus, these studies were mainly performed on a subgroup not suffering from relevant co-morbidities and being in good performance status.

In recent years, the need to investigate real life populations and the common practice of dose reduction has been recognized by the scientific community. However, (retrospective) studies investigating the effects of dose reduction in a palliative setting have been published but are still sparse. In 2001, a retrospective analysis of

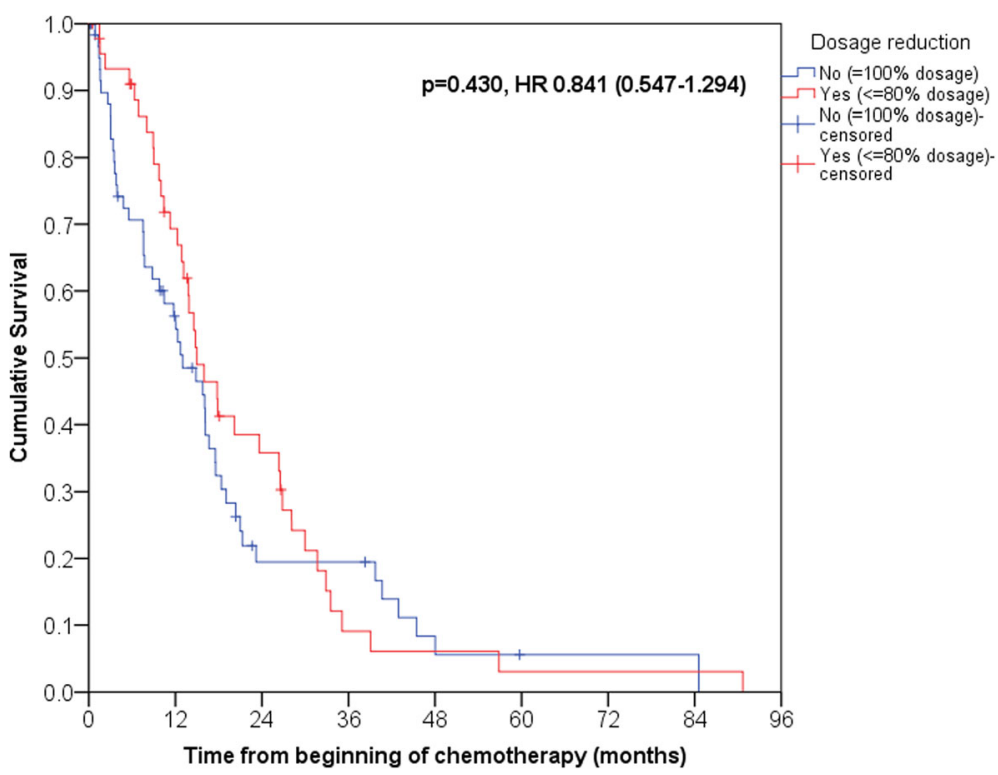

Fig. 1 Kaplan Meier analysis of survival in patients with reduced and full dosage of standard chemotherapy back bone (FOLFIRI, FOLFOX). Survival analysis showed no difference in survival ( $p=0.430$, Log Rank) 
Table 4 Results of univariable and multivariable Cox-regression for survival according to dosage reduction

\begin{tabular}{|c|c|c|c|c|c|}
\hline & & & & $95 \% \mathrm{Cl}$ & \\
\hline & & $p$ value & Hazard ratio & Lower & Upper \\
\hline Univariable Cox-regression & & & & & \\
\hline Dosage reduction & No & & 1.000 & & \\
\hline & Yes & .431 & .841 & .547 & 1.294 \\
\hline Multivariable Cox-regression & & & & & \\
\hline Dosage reduction & No & & 1.000 & & \\
\hline & Yes & .600 & .861 & .492 & 1.506 \\
\hline Age & continuous & .118 & .981 & .958 & 1.005 \\
\hline Sex & Male & & 1.000 & & \\
\hline & Female & .446 & 1.264 & .692 & 2.310 \\
\hline T stage & T3 & & 1.000 & & \\
\hline & T4 & .229 & 1.541 & .761 & 3.117 \\
\hline & TX & .890 & .916 & .265 & 3.170 \\
\hline $\mathrm{N}$ stage & No & & 1.000 & & \\
\hline & N1 & .981 & .988 & .345 & 2.824 \\
\hline & N2 & .713 & 1.225 & .415 & 3.615 \\
\hline & NX & .927 & .922 & .161 & 5.266 \\
\hline Grading & G2 & & 1.000 & & \\
\hline & G3 & .302 & 1.354 & .761 & 2.407 \\
\hline & GX & .458 & .576 & .134 & 2.474 \\
\hline L stage & LO & & 1.000 & & \\
\hline & L1 & .726 & 1.215 & .408 & 3.620 \\
\hline & LX & .289 & .469 & .116 & 1.901 \\
\hline V stage & Vo & & 1.000 & & \\
\hline & V1 & .872 & .920 & .334 & 2.531 \\
\hline & $V X$ & .164 & 2.620 & .675 & 10.165 \\
\hline Charlson Score & 6 & & 1.000 & & \\
\hline & 7 & .750 & .892 & .443 & 1.797 \\
\hline & 8 & .909 & .954 & .426 & 2.138 \\
\hline & 9 & .609 & 1.605 & .262 & 9.822 \\
\hline Oncological resection/Surgery & No & & 1.000 & & \\
\hline & Yes & .227 & .483 & .149 & 1.570 \\
\hline No of CTX lines & continuous & .797 & 1.053 & .712 & 1.556 \\
\hline Biological CTX & No & & 1.000 & & \\
\hline & Yes & .178 & .663 & .364 & 1.207 \\
\hline CTX regimen & FOLFOX-> FOLFIRI & & 1.000 & & \\
\hline & FOLFIRI- > FOLFOX & .691 & 1.177 & .527 & 2.627 \\
\hline & FOLFOX & .001 & 3.601 & 1.656 & 7.827 \\
\hline & FOLFIRI & .921 & .945 & .309 & 2.892 \\
\hline
\end{tabular}

patients with Stage II-III colon cancer demonstrated that in an adjuvant setting 5-FU based chemotherapy may be safely administered in the elderly, but this study did not elaborate the dose reduction needed [12]. In 2011 Langley et al. published the Focus II study, a first randomized controlled trial including only the frail and old patients with colorectal cancer. This study incorporated primary dose reduction as the standard treatment for all treatment arms. However, it was not designed to investigate whether dose 
Table 5 Subgroup analysis. Comparison of the survival after dose reduction versus full dosage in different subgroups analyzed by Kaplan-Meier procedure and Log-Rank test (Chemotherapy (CTX))

\begin{tabular}{|c|c|c|c|c|}
\hline & & Dosage redu & & Log-rank \\
\hline & & Yes ( $\leq 80 \%)$ & No (100\%) & \\
\hline & & Mean (Media & ths & $p$-value \\
\hline Age & $<65$ & $22.0(14.6)$ & $17.1(12.0)$ & .170 \\
\hline & $\geq 65$ & $18.8(14.9)$ & $21.2(15.8)$ & .764 \\
\hline Sex & Male & $23.5(16.0)$ & $18.6(14.9)$ & .339 \\
\hline & Female & $17.2(13.1)$ & $17.4(12.7)$ & .170 \\
\hline T stage & $\mathrm{T} 3$ & $27.8(26.4)$ & $25.8(14.9)$ & .525 \\
\hline & T4 & $15.6(13.8)$ & $14.1(12.7)$ & .960 \\
\hline & Tx & $24.3(10.4)$ & $14.1(7.7)$ & .273 \\
\hline N stage & No & 25.9 (23.6) & 30.5 (14.9) & .851 \\
\hline & N1 & 20.7 (13.9) & $24.1(12.3)$ & .669 \\
\hline & N2 & $21.3(26.4)$ & $13.3(16.7)$ & .024 \\
\hline & $N x$ & $20.4(16.0)$ & $13.2(7.7)$ & .294 \\
\hline Grading & G2 & $24.1(17.8)$ & $22.0(16.1)$ & .570 \\
\hline & G3 & $17.5(14.5)$ & $10.6(10.4)$ & .062 \\
\hline & Gx & $26.8(26.8)$ & $25.5(3.0)$ & .808 \\
\hline L stage & LO & $19.7(17.8)$ & $30.7(21.0)$ & .541 \\
\hline & L1 & $23.3(13.9)$ & 16.9 (17.5) & .485 \\
\hline & $L x$ & $18.1(14.8)$ & $16.3(12.0)$ & .561 \\
\hline V stage & V0 & $28.8(23.6)$ & $26.9(21.0)$ & .828 \\
\hline & V1 & $20.3(13.9)$ & 15.7 (16.7) & .311 \\
\hline & $V x$ & 16.9 (14.5) & 15.9 (11.8) & .671 \\
\hline Charlson Score for Comorbidities & 6 & $20.6(14.8)$ & $19.6(12.0)$ & .424 \\
\hline & $7-10$ & $23.3(15.0)$ & $19.6(16.1)$ & .632 \\
\hline Oncological resection/Surgery & Yes & $22.1(14.9)$ & $23.8(17.5)$ & .997 \\
\hline & No & $19.6(12.9)$ & $12.9(7.6)$ & .301 \\
\hline No. of CTX lines & 1 & $10.1(10.4)$ & $8.4(3.0)$ & .565 \\
\hline & 2 & $23.1(17.9)$ & $23.5(15.8)$ & .896 \\
\hline & 3 & $24.7(23.6)$ & $16.6(12.3)$ & .238 \\
\hline Biological CTX & Yes & $29.4(26.8)$ & $23.5(11.8)$ & .371 \\
\hline & No & $13.2(13.1)$ & $14.6(14.9)$ & .823 \\
\hline $\mathrm{CTX}$ regimen sequence & FOLFOX-> FOLFIRI & $22.0(20.2)$ & $26.5(17.5)$ & .425 \\
\hline & FOLFIRI- > FOLFOX & $12.3(12.3)$ & $16.4(14.9)$ & .631 \\
\hline & FOLFOX & $9.7(12.9)$ & $7.7(3.6)$ & .829 \\
\hline & FOLFIRI & $62.3(90.7)$ & $13.0(3.9)$ & .300 \\
\hline
\end{tabular}

reduction itself could be performed if required without affecting PFS or OS [13].

In order to evaluate whether dose reduction has an effect on survival in patients with advanced colorectal cancer and suffering from side effects under standard treatment dose, we performed this retrospective analysis of such patients in our outpatient clinic. In our current study, we chose a cutoff for dose reduction of $80 \%$ since in our experience a dose reduction to $80 \%$ is a commonly performed reduction in case of adverse reactions. Since only very few patients received further dose reduction $(n=4)$, we chose not to include these patients as a separate group with dose reduction of $\leq 50 \%$ but rather included these patients in the group of patients with reduction of chemotherapy dosage. Thus, the overall average dose reduction in this patient group was even more than 20\%. In these patients, a dose reduction was applied to the 
majority of cycles (72\%), emphasizing that this group predominantly received a reduced chemotherapy dosage throughout the course of therapy.

In daily clinical routine, clinicians are more likely to reduce the dose of elderly patients. Therefore, one might assume that in the elderly ( $>65$ years) in our collective dose reduction would be more common. However, dose reduction was evenly distributed among younger and elderly patients and thus independent of age. Also, additional subgroup analysis (T-stage, $\mathrm{N}$-stage, $\mathrm{M}$-stage, gender, and chemotherapy regimen) showed an even distribution of dose reduction.

Since clinical trials showed the effectiveness of per protocol chemotherapy, in theory a reduced dosage of chemotherapy would be expected to affect survival, which has also been confirmed by several publications for other entities [9]. It is therefore common belief, that dose reduction should be avoided. Additionally, clinicians choose a dose reduction often due to symptomrelated causes or deterioration of laboratory findings. Many of these reasons (recurring neutropenia, deterioration of general condition) are associated with a poor clinical outcome. Therefore, one might assume, that dose reduction is more common in patients with clinical features suggesting a poor prognosis. Interestingly, statistical analysis of our data showed that a moderate dose reduction does not affect survival significantly.

In a recently published manuscript, the influence of the relative dose intensity (RDI, of adjuvant 5FU and Oxaliplatin combination treatment in veterans with Stage III colon cancer had been further investigated. Aspinalli et al. showed that a major reduction of RDI $(<70 \%)$ to be associated with worse overall survival in this patient group [14]. In addition to the bias of the patient group which consisted mainly of male veterans, there was a bias towards dose reduction in the frail and elderly. In comparison with our study also the magnitude of dose reduction was more pronounced.

When Oxaliplatin was introduced to the treatment of $\mathrm{CRC}$, a retrospective analysis of three phase II studies of pretreated colorectal cancer showed that higher dose intensity leads to improved survival [15]. In contrast to our study, these study populations had been pretreated and in two out of the three studies patients' inclusion age was limited to younger patients. In another more recent study, Nakayama et al. showed that a dose reduction in metastasized CRC led to poorer survival of the respective (Irinotecan) patient group. A comparison with our study population is difficult, since per protocol Irinotecan dosage in Japan is already $12 \%$ lower than in the western countries and further dose reduction adds up to even more pronounced dose reductions. For the patient group receiving Oxaliplatin only PFS was significantly associated to RDI [16].
Nevertheless, in clinical practice, patients often experience side effects and need dose reduction. For these patients, our data in treatment-naive patients suffering from stage IV colorectal cancer, suggest that a moderate dose reduction does not necessarily result in less efficacy. Until now, only limited data were reported on this issue, which we believe to be of high clinical relevance. Thus, we suggest further randomized studies potentially leading to more personalized treatment strategies depending on tolerance of treatment and co-morbidities and a more side effect oriented approach on chemotherapy dosing.

\section{Conclusion}

In our group of patients with colorectal cancer treated in a palliative setting, the need for a moderate reduction of chemotherapy due to side effects has no measurable effect on survival. This may be in part due to better adaption to interindividual pharmacokinetics and to a longer treatment of patients with reduced chemotherapy dosage if side effects cause dose reduction.

\section{Additional file}

Additional file 1: Table S1. Summary of comorbidities. (DOCX 13 kb)

\section{Acknowledgements}

We greatly appreciate all the authors for their endeavor.

Availability of data and materials

The datasets used and/or analysed during the current study are available from the corresponding author on reasonable request.

\section{Comment}

Part of this study was presented at the ASCO Gl Meeting 2017 San Francisco. Permission was obtained for final publication [17].

\section{Authors' contributions}

SM, MG, AT carried out the primary data analysis. SM, AT, SFF, CS, HJS, CO, $E S, P W, M V, W H, M E, M R, P F, M K S$ were responsible for the treatment of patients with CRC at the Regensburg University Medical Center. SM, AT, MG, PW and HJS helped to draft the manuscript. All authors read and approved the final manuscript.

Ethics approval and consent to participate

Due to the analysis of data from a clinical cancer registry, no ethics approval was necessary. This was confirmed by the Ethics Committee at the Regensburg University, Regensburg, Germany.

Competing interests

The authors declare that they have no competing interest.

\section{Publisher's Note}

Springer Nature remains neutral with regard to jurisdictional claims in published maps and institutional affiliations. 


\section{Author details}

'Department of Internal Medicine I, University Hospital Regensburg, Franz-Josef-Strauss Allee 11, 95053 Regensburg, Germany. ${ }^{2}$ Cancer Center, Institute for quality assurance and health services research, University of Regensburg, Regensburg, Germany. ${ }^{3}$ Medical Informatics Unit, University Hospital Regensburg, Regensburg, Germany. ${ }^{4}$ Department of Surgery, University Hospital Regensburg, Regensburg, Germany. ${ }^{5}$ Department of Radiology, University Hospital Regensburg, Regensburg, Germany. ${ }^{6}$ Department of Internal Medicine III, University Hospital Regensburg, Regensburg, Germany. ${ }^{7}$ Department of Pathology, University Hospital Regensburg, Regensburg, Germany. ${ }^{8}$ MedicDAT GmbH, Regensburg, Germany.

Received: 23 May 2016 Accepted: 16 April 2018

Published online: 23 April 2018

\section{References}

1. Siegel R, Desantis C, Jemal A. Colorectal cancer statistics, 2014. CA Cancer J Clin. 2014;64:104-17.

2. Kelly H, Goldberg RM. Systemic therapy for metastatic colorectal cancer: current options, current evidence. J Clin Oncol. 2005;23:4553-60.

3. Braun MS, Seymour MT. Balancing the efficacy and toxicity of chemotherapy in colorectal cancer. Ther Adv Med Oncol. 2011;3:43-52.

4. Hutchins LF, Unger JM, Crowley JJ, et al. Underrepresentation of patients 65 years of age or older in cancer-treatment trials. N Engl J Med. 1999:341:2061-7.

5. Kordatou Z, Kountourakis P, Papamichael D. Treatment of older patients with colorectal cancer: a perspective review. Ther Adv Med Oncol. 2014;6:128-40

6. Hurria A, Dale W, Mooney $M$, et al. Designing therapeutic clinical trials for older and frail adults with cancer: U13 conference recommendations. J Clin Oncol. 2014;32:2587-94.

7. Leo S, Accettura C, Gnoni A, et al. Systemic treatment of gastrointestinal cancer in elderly patients. J Gastrointest Cancer. 2013;44:22-32.

8. Bluhm M, Connell CM, Janz N, et al. Oncologists' End of Life Treatment Decisions: How Much Does Patient Age Matter? J Appl Gerontol. 2015;36(4): 416-40.

9. Havrilesky $L$, Reiner M, Morrow PK, et al. A review of relative dose intensity and survival in patients with metastatic solid tumors. Crit Rev Oncol Hematol. 2015;93:203-10.

10. Charlson ME, Pompei $\mathrm{P}$, Ales KL, et al. A new method of classifying prognostic comorbidity in longitudinal studies: development and validation. J Chronic Dis. 1987;40:373-83.

11. Reng C, Fest P, Mackensen A, et al. OnkoDAT - Unterstützung der Chemotherapieplanung. PraxisComputer. 2002;18:10-2.

12. Fata F, Mirza A, Craig G, et al. Efficacy and toxicity of adjuvant chemotherapy in elderly patients with colon carcinoma: a 10-year experience of the Geisinger Medical Center. Cancer. 2002;94:1931-8.

13. Seymour MT, Thompson LC, Wasan HS, et al. Chemotherapy options in elderly and frail patients with metastatic colorectal cancer (MRC FOCUS2): an open-label, randomised factorial trial. Lancet. 2011;377:1749-59.

14. Aspinall SL, Good CB, Zhao X, et al. Adjuvant chemotherapy for stage III colon cancer: relative dose intensity and survival among veterans. BMC Cancer. 2015;15:62.

15. Maindrault-Goebel F, de Gramont A, Louvet C, et al. Evaluation of oxaliplatin dose intensity in bimonthly leucovorin and 48-hour 5-fluorouracil continuous infusion regimens (FOLFOX) in pretreated metastatic colorectal cancer. Oncology Multidisciplinary Research Group (GERCOR). Ann Oncol. 2000;11:1477-83.

16. Nakayama G, Tanaka C, Uehara K, et al. The impact of dose/time modification in irinotecan- and oxaliplatin-based chemotherapies on outcomes in metastatic colorectal cancer. Cancer Chemother Pharmacol. 2014;73:847-55.

17. Munker S. Chemotherapy for metastatic colon cancer: Effect on survival when the dose is reduced due to side effects. J Clin Oncol. 2017;35

\section{Ready to submit your research? Choose BMC and benefit from:}

- fast, convenient online submission

- thorough peer review by experienced researchers in your field

- rapid publication on acceptance

- support for research data, including large and complex data types

- gold Open Access which fosters wider collaboration and increased citations

- maximum visibility for your research: over $100 \mathrm{M}$ website views per year

At BMC, research is always in progress.

Learn more biomedcentral.com/submissions 